genotype. Statistical analysis was performed on carriers of the rs45455403 genotype, to determine its predictor value for specific cognitive features. Over 80 neurological characteristics were explored for significant differences between the wild-type and the rs 45455403 polymorphism carriers. Results: Observed cognitive differences between the wild-type and the rs45455403 polymorphism carriers included processing speed, naming, word-finding difficulties, problems with verbal repetition, reading, judgment, following instructions, sense of direction, anxiety, and insight to memory loss. These were observed even in subjects without overt symptoms of dementia. An additional 100 specimens from African-American individuals with careful cognitive profiling are currently undergoing genetic analysis. Conclusions: Studying the cognitive spectrum of patients carrying the rs45455403 genotype can help identify the earliest manifestations of Alzheimer's disease in this African-American sub-group. Clinical characterization of this genetic subgroup may assist the identification of at-risk individuals or the clinical biomarkers for early diagnosis and/or progression. Current research suggests that early disease-modifying interventions or prevention strategies are highly effective for at-risk patients.

\section{P1-058 THE HSP90 CO-CHAPERONE FKBP51 PRODUCES NEUROTOXIC TAU OLIGOMERS: IMPLICATION FOR AGING AND ALZHEIMER'S DISEASE}

Laura Blair $^{1}$, Bryce Nordhues ${ }^{1}$, Shannon Hill ${ }^{1}$, K. Matthew Scaglione ${ }^{2}$, John O'Leary ${ }^{1}$, Leonid Breydo ${ }^{1}$, Zhang Bo ${ }^{1}$, Pengfei Li $^{1}$, Lily Wang ${ }^{1}$, Carl Cotman ${ }^{3}$, Henry Paulson ${ }^{2}$, Martin Muschol ${ }^{1}$, Vladimir Uversky ${ }^{1}$, Torsten Klengel ${ }^{4}$, Elisabeth Binder ${ }^{4}$, Rakez Kayed ${ }^{5}$, Nicole Berchtold ${ }^{6}$, Todd Golde ${ }^{7}$, Chad Dickey ${ }^{1},{ }^{1}$ University of South Florida, Tampa, Florida, United States; ${ }^{2}$ University of Michigan, Ann Arbor, Michigan, United States; ${ }^{3}$ UCI MIND, Irvine, California, United States; ${ }^{4}$ Max Planck Institute for Psychiatry, München, Germany; ${ }^{5}$ University of Texas Medical Branch, Galveston, Texas, United States; ${ }^{6}$ University of California, Irvine, Irvine, California, United States; ${ }^{7}$ University of Florida, Gainesville, Florida, United States.Contact e-mail: lblair@health.usf.edu

Background: FK506 binding protein 51kDa (FKBP51/FKBP5) is a heat shock protein $90 \mathrm{kDa}$ (Hsp90) co-chaperone known to regulated tau biology. Tau is a microtubule associated protein that becomes hyperphosphorylated and forms intracellular neurofibullary tangles in the brain leading to Alzheimer's disease (AD). Neuronal death in AD exceeds the number of tau tangles, which suggests other toxic tau species are involved in neurodegeneration. Methods: Biochemical analysis of recombinant protein was performed using circular dichroism (CD), dynamic light scattering (DLS), and atomic force microscopy (AFM). Stereotaxic injections were used to deliver FKBP5 tagged AAV particles into the hippocampus of rTg4510 tau mice. Immunohistochemistry was performed on brain tissue to evaluate tau protein levels. Human mRNA and DNA were evaluated for FKBP5 gene expression and methylation respectively. Results: In vitro recombinant tau assays reveal that FKBP51 works in coordination with Hsp90 to preserve tau from proteasomal degradation in an oligomeric state. In vivo, FKBP5 overexpression preserves neurotoxic, oligomeric tau. In the human brain, FKBP5 mRNA expression levels dramatically increase while FKBP5 DNA methylation decreases with age. In AD, FKBP5 mRNA expression levels positively correlate with Braak staging, and inversely correlate with FKBP5 methylation. Conclusions: FKBP51 is able to prevent tau clearance by the proteasome, preserving tau in an oligomeric form that is neurotoxic. FKBP51 is up-regulated with aging and increases further in AD. Overall these data suggest high FKBP51 expression creates a proteostatic imbalance leading to tau toxicity. Therefore, FKBP51 may accelerate AD onset and progression, suggesting that strategies targeting this Hsp90 co-chaperone could be therapeutically relevant for $\mathrm{AD}$ and other tauopathies.

\section{P1-059 WHOLE GENOME SEQUENCING IN AN UNRESOLVED ALZHEIMER'S DISEASE FAMILY LINKED TO 7 Q36}

Rita Cacace ${ }^{1}$, Elise Cuyvers ${ }^{1}$, Karolien Bettens ${ }^{1}$, Caroline Van Cauwenberghe ${ }^{1}$, Jasper Van Dongen ${ }^{1}$, Marc Cruts ${ }^{1}$, Kristel Sleegers ${ }^{1}$,
Christine Van Broeckhoven ${ }^{1},{ }^{1}$ VIB and Institute Born-Bunge, University of Antwerp, Antwerp, Belgium. Contact e-mail: rita.cacace@molgen.vib-ua. be

Background: We are investigating a 3-generation autosomal dominant family with a clinical diagnosis compatible with Alzheimer disease (AD) (onset $66.8 \pm 7.4$ years $(\mathrm{N}=13$; age range $47-77$ years). In a whole-genome linkage study, we linked the family to a genomic region of $5.44 \mathrm{Mb}$ at $7 \mathrm{q} 36$ (Rademakers et al. AJHG, 2005). Exonic sequencing of 29 genes as well as dosage analysis using array-based comparative genomic hybridization (aCGH) identified one synonymous mutation in PAXIP1. However, we could not provide convincing evidence for a functional role of PAXIP1 in disease pathology leaving the possibility that another mutation at this locus might explain the observed linkage. Therefore, we used next generation sequencing (NGS) technology to re-examine the linked candidate region at $7 \mathrm{q} 36$. Methods: Four distantly related patients were selected and whole genome sequencing (WGS) was performed by Complete Genomics, Inc. The WGS data was analyzed using an in-house developed software tool GenomeComb. Sequenom MassARRAY $®$ and Sanger sequencing were used for validation, for segregation in the family and for screening of control individuals $(\mathrm{N}=1029)$ and $\mathrm{AD}$ patients $(\mathrm{N}=1263)$. Results: The sequence of the linked region was extracted from the WGS data and compared between the 4 patients. To select truly called, novel or rare variations $(\mathrm{MAF}<1 \%)$, we applied quality and frequency filters. We identified 93 heterozygous variations that were shared between the 4 patients of which 35 were segregating within the family including the one in PAXIP1. After testing the 35 segregating variations in the control cohort only 4 variations remained for further analyses. Interestingly, is that 3 of these 4 variations are located in the same gene and cluster in the same intron. The remaining one is an intergenic variation. All 4 variations affect sequences of open chromatin and involve putative transcription factor binding sites (ENCODE project data). Conclusions: The WGS approach is a promising tool to identify linked variations in non-coding regions in an unresolved locus identified in an extended AD family. The clustering of the variations is indicative for a putative pathogenic role by influencing the expression and/or regulation of nearby genes.

\section{P1-060 THE INFLUENCE OF GENETIC VARIANTS IN SORL1 GENE ON THE MANIFESTATION OF ALZHEIMER'S DISEASE}

Eva Louwersheimer ${ }^{1}$, Carlos Cruchaga ${ }^{2}$, Pieter Jelle Visser ${ }^{3}$, Henne Holstege ${ }^{4}$, Philip Scheltens ${ }^{4}$, John Van Swieten ${ }^{5}$, Wiesje Van Der Flier ${ }^{6},{ }^{1}$ Alzheimer Center, VU University Medical Center, Amsterdam, Netherlands; ${ }^{2}$ Washington University School of Medicine, Saint Louis, Missouri, United States; ${ }^{3}$ VUMC, Amsterdam, Netherlands; ${ }^{4}$ VU University Medical Center, Amsterdam, Netherlands; ${ }^{5}$ Erasmus University Medical Center, Rotterdam, Netherlands; ${ }^{6} \mathrm{VU}$ University Medical Center, Amsterdam, Netherlands.Contact e-mail: e.louwersheimer@vumc.nl

Background: Alzheimer's disease (AD) is a multigenetic disorder. A large number of risk variants have been discovered influencing the risk of sporadic $\mathrm{AD}$, among which $\mathrm{APOE}$ is the strongest risk factor. Several studies found a significant association between single nucleotide polymorphisms (SNPs) in the sortilin-related receptor (SORL1) gene on chromosome 11 and AD. SORL1 plays an important role in the intracellular trafficking of APP and is also a low-density lipoprotein receptor. We evaluated how SORL1 SNPs influence manifestation of disease in terms of cognitive profile, hippocampal atrophy and cerebrospinal fluid (CSF) biomarkers in $\mathrm{AD}$ patients from the Alzheimer's Disease Neuroimaging Initiative (ADNI). Methods: 83 caucasian patients (non-hispanic and non-latino) with probable AD were included from the ADNI. We selected 8 SNPs from the genotyped data coming from the ADNI database and imputed 11 SNPs. All SNPs were dichotomized based on presence of at least one risk allele. As dependent variables we used measures of cognitive performance (Mini Mental State Exame (MMSE) and Alzheimer's Disease Assessment Scale (ADAS)), hippocampal volumes and the CSF biomarkers Amyloid-beta142 (abeta), total tau and tau phosporylated at threonine 181 (ptau). First we used univariate linear regression to assess associations between SNPs 